\title{
Workshop: regulatory affairs
}

\author{
Sue Barlow* \\ 8 Harrington Road, Brighton, BN1 6RE, UK
}

Polyols: Warning labels: Laxation: EU legislation

The workshop addressed the main issues influencing the regulation of low-digestible carbohydrates (LDCs), which include the selective use of warning labels on laxation, the soundness of research underpinning such warnings, and the restrictions on the use of polyol sweeteners in the EU. The workshop considered whether current national and international regulations and standards covering the use and labelling of LDCs were appropriate and if not, whether current scientific evidence supported the case for regulatory change. It was discussed if any specific recommendations concerning regulatory affairs could be made on the basis of current scientific knowledge regarding benefits, safety and tolerance. It was also considered what further research might help clarify regulatory issues. The workshop noted several areas of legislation that raise problems for both consumers and industry, namely: the singling out of polyols used as sweeteners to carry special warning labels; the variation in warning label requirements between different regions; restrictions on the use of polyol sweeteners in the EU to energy-reduced foods and foods with no added sugar; lack of agreement within the EU on use of health and physiological claims.

\section{Warning labels and laxation}

Mandatory warning labels drawing attention to the possibility of laxation are required under several national and international regulations. However, there is little consistency between different countries in either the level(s) triggering mandatory labelling or the selection of which polyols should be labelled. The Codex rule (Codex, 1991 ) is very general, requiring labelling if sugar alcohols are present in a food in quantities $>20 \mathrm{~g}$. In the USA, labelling is required only in the case of certain LDCs (mannitol $>20 \mathrm{~g} /$ day, sorbitol $>50 \mathrm{~g} /$ day, polydextrose $>15 \mathrm{~g} /$ serving) (US Code of Federal Regulations, CFR 180.25; CFR 184.1835; CFR 172.841). In the EU labelling is required when the content of any polyol sweetener in a food is $>10 \%$ (EC, 1996). It was also noted that there are proposals to introduce maximum permissible amounts of polyols in foods before an advisory label is required (either $10 \mathrm{~g}$ or $25 \mathrm{~g} / 100 \mathrm{~g}$ of product, depending on the polyol) in Australia.
It was felt that the different trigger levels and different selectivity in LDCs requiring a warning was a reflection of the absence of any firm scientific base for laxation warnings. Evidence presented earlier in the symposium had illustrated the paucity of appropriate human studies to indicate population thresholds for laxation for individual polyols. The influence of other dietary components and variations in gut flora has not been adequately taken into account. A number of studies have utilised vehicles of administration for polyols, such as jelly, which maximise the potential to cause laxation and do not reflect the normal situation of consumption within a food matrix. Although potential high consumers, such as diabetics, can be identified, little attempt has been made to study and inform potentially sensitive subgroups in the population (e.g. those with bowel disorders, such as irritable bowel syndrome or coeliac disease). The utility of warning labels on individual products was questioned given that laxation depends on overall daily intakes of LDCs and consumers have difficulty in summing their daily intake.

It was further noted that other poorly absorbed foods that can cause laxation, if amounts consumed exceed the physiological capacity of the gut to tolerate them, were not so labelled. It was recognised that this situation has perhaps arisen in the EU because polyols fall under the Sweeteners Directive of the EU (1994) additives regulations and thus require prior approval, whereas other low digestible carbohydrates occurring naturally in the diet or used as food ingredients do not. The overall situation is unhelpful to consumers and impedes willingness by industry to develop new and potentially beneficial products.

\section{Laxative thresholds}

It was acknowledged that laxation due to consumption of LDCs in excess of what an individual can tolerate is transiently disturbing and discomfiting and could lead to an elevated risk of misdiagnosis and so inappropriate medical intervention, if the cause was not recognised. However, it was felt that this is an issue of tolerance rather than safety, and, as such, it warrants advice rather than warnings. Such advice should be in relation to the overall diet rather than individual foods; not only polyol consumption but also 
consumption of LDCs other than polyols was relevant. The scientific basis for setting laxation thresholds for individual polyols was poor and variations between individuals in tolerance of LDCs may be considerable.

In view of these considerations, it was proposed that consumer advice and education about tolerance to LDCs in general, alongside information about potential benefits, would be far more helpful to consumers than warnings on individual products containing polyol sweeteners. At the same time, it was agreed that manufacturers have a responsibility to ensure they do not market polyol-containing products, such as drinks and gum or jelly confectionery, that might easily be consumed in high amounts. The present lack of agreement within the EU, and consequent lack of legislation, about the appropriate use of health or physiological claims is another impediment to consumer information and education about the beneficial effects of LDCs. At present only Japan has an approval system for functional foods offering physiological benefit and/or health benefits.

\section{EU legislation and polyol sweeteners}

The EU legislation restricting use of polyol sweeteners to energy-reduced foods and to foods with no added sugar (EU, 1994) raises several problems. The definitions of such foods are not widely understood and definitions differ between the EU and other countries. The prohibition on utilising sugar/polyol combinations prevents the development of foods which might combine the two types of sweetener to yield products with acceptable sweetness, in which tolerance is not a problem, and which still provide potential benefits. It was felt that lifting of these restrictions would be beneficial both to consumers and to the industry.

\section{Further research}

In considering what research might help to clarify some of the regulatory problems identified above, it was agreed that recent work illustrated that a simple approach to identifying thresholds for laxation for specific polyols was no longer appropriate. This casts the validity and utility of the current mandatory warnings into some doubt. To develop better advice for consumers, more attention should be directed towards studies which take the overall daily diet into account, which will help define the dietary conditions likely to minimise tolerance problems, and which help identify potentially sensitive subgroups.

Although regulatory systems have been developed to protect the consumer from potential adverse effects and unsubstantiated medicinal claims, consideration of the need to have in place regulatory systems which enable consumers to be better informed about the potential benefits of foods, beyond their straightforward nutritional content, is lagging behind. For LDCs to benefit from any such regulatory changes in the future, further research should be encouraged to build on the considerable progress which has already been made in identifying the potential beneficial effects of LDCs in common conditions such as obesity, diabetes, hyperlipidaemia, hypertension and on bowel physiology.

\section{References}

Codex (1991) Codex standard for formula foods for use in weight control diets. Codex Standard 181.

EU (1994) European Parliament and Council Directive 94/35/EC of 30 June 1994 on sweeteners for use in foodstuffs. Official Journal of the European Communities No. L 237/3-12. 10.9.94.

European Communities (1996) Council Directive 96/21/EC of 29 March 1996 amending Commission Directive 94/54/EC concerning the compulsory indication on the labelling of certain foodstuffs of particulars other than those provided for in Directive 79/112/EEC. Official Journal of the European Communities No. L 88, 5.4.96, p. 5, Luxembourg.

US Code of Federal Regulations, Washington DC: 21 CFR 180.25 .

US Code of Federal Regulations, Washington DC: 21 CFR 184.1835 .

US Code of Federal Regulations, Washington DC: 21 CFR 172.841 . 